\title{
Age-specific onset and distribution of the natural anticoagulant deficiency in pediatric thromboembolism
}

\author{
Masako Ichiyama', Shouichi Ohga², Masayuki Ochiai', Koichi Tanaka', Yuka Matsunaga', Takeshi Kusuda', Hirosuke Inoue', \\ Masataka Ishimura ${ }^{3}$, Tomohito Takimoto ${ }^{3}$, Yui Koga ${ }^{4}$, Taeko Hotta ${ }^{4}$, Dongchon Kang ${ }^{4}$ and Toshiro Hara ${ }^{1,3}$
}

BACKGROUND: The early diagnosis of inherited thrombophilia in children is challenging because of the rarity and hemostatic maturation.

METHODS: We explored protein C (PC), protein S (PS), and antithrombin (AT) deficiencies in 306 thromboembolic patients aged $\leq 20 \mathrm{y}$ using the screening of plasma activity and genetic analysis.

RESULTS: Reduced activities were determined in 122 patients (40\%). Low PC patients were most frequently found in the lowest age group (0-2 y, 45\%), while low PS or low AT patients were found in the highest age group (16-20 y; PS: 30\% and AT: 20\%). Genetic study was completed in 62 patients having no other causes of thromboembolism. Mutations were determined in 18 patients (8 PC, 8 PS, and 2 AT genes). Six of eight patients with $P C$ gene mutation were found in age $0-2 y$ (75\%), while six of eight patients with PS gene mutation were in 7-20 y. Two AT gene-mutated patients were older than $4 \mathrm{y}$. Four PC-deficient and two PS-deficient patients carried compound heterozygous mutations. All but one PC gene-mutated patient suffered from intracranial thromboembolism, while PS/AT gene-mutated patients mostly developed extracranial venous thromboembolism.

CONCLUSION: Stroke in low PC infants and deep vein thrombosis in low PS/AT school age children could be targeted for genetic screening of pediatric thrombophilias.

$\mathbf{T}$ hromboembolism is a multifactorial disease involving genetic predispositions, underlying disorders, and varied triggers including infection or injury. Vascular, circulatory, and hemostatic conditions are distinctively associated with the development of arterial and venous thromboses. Thromboembolism, formerly recognized as a rare event in children (1), has been increasingly diagnosed as a complication of sepsis, cancer, cardiovascular disease, and therapyrelated events. Recent advances in the intensive cares, cardiac surgery, and transplantation medicine along with the imaging diagnosis may contribute to the increased number of pediatric patients with thrombosis $(2,3)$. The established genetic risks of venous thromboembolism (VTE) include protein C (PC), protein S (PS), and antithrombin (AT) deficiency, as well as factor V G1691A (FVL) and prothrombin G20210A (FII) variants (4). The high incidence of VTE in Caucasians (5) is explained by the fact that FVL and FII G20210A carriers were found in $20-60 \%$ of adult VTE patients in Caucasian but not Asian ancestries (6-9). PC, PS, and AT deficiencies share the lower prevalence than FVL and/or FII G20210A carriers, but the higher risk of the first and recurrent VTE than the other thrombophilias. On the other hand, the diagnosis is challenging during infancy and early childhood, because the references of increasing anticoagulant activity range widely until adolescence $(10,11)$. The true effect of the natural anticoagulant deficiencies on the development of pediatric thromboembolism remains elusive because of the rare occurrence and the elaborative genetic screenings.

To clarify the clinical impact of the inherited deficiency of natural anticoagulant on children, we conducted the genetic analysis of PC, PS, and AT deficiency after the screening of each activity for pediatric patients with thromboembolism over $20 \mathrm{y}$ in a single institution. The results demonstrated the distinct presentation of PC and PS deficiencies in infants and children, respectively, reflecting the genotype of adult Japanese patients.

\section{RESULTS}

\section{Distribution of Thromboembolic Patients}

Of 306 patients aged $\leq 20$ y (male: female 1:1.14), 186 suffered from intracranial lesions including ischemic and/or hemorrhagic strokes. The extracranial lesions consisted of renal vein thrombosis $(n=7)$, purpura fulminans $(n=3)$, deep vein thrombosis in the leg $(n=15)$, and pulmonary thromboembolism $(n=9)$. Patients younger than 3 y were $27 \%$ of all patients. The largest age group was $<1 \mathrm{y}$ of age $(n=50,16.3 \%)$, and the number of other age groups were similar (median: 12, ranging: $9-17(3.0-5.5 \%))$.

\section{PC, PS, and AT Activities in Patients}

A total of 122 patients had the reduced activity of either anticoagulant; 95 with low PC (31\%), 48 with low PS (16\%), and 20 patients with low AT activity (7\%; Figure 1). Low PC patients

${ }^{1}$ Comprehensive Maternity and Perinatal Care Center, Kyushu University Hospital, Fukuoka, Japan; ${ }^{2}$ Department of Pediatrics, Yamaguchi University Graduate School of Medicine, Ube, Japan; ${ }^{3}$ Department of Pediatrics, Graduate School of Medical Sciences, Kyushu University, Fukuoka, Japan; ${ }^{4}$ Department of Clinical Chemistry and Laboratory Medicine, Kyushu University Hospital, Fukuoka, Japan. Correspondence: Shouichi Ohga (ohgas@yamaguchi-u.ac.jp)

Received 5 March 2015; accepted 29 June 2015; advance online publication 14 October 2015. doi:10.1038/pr.2015.180 
were most frequently determined in the lowest age group (0-2 $\mathrm{y}, 45 \%)$, while low PS or low AT patients were frequently found in the highest age group (16-20 y; PS: $30 \%$ and AT: $20 \%)$. The number of patients with low activity in age groups are shown in Figure 2. The proportions of low PC patients were higher than those of low PS or AT patients in age $0-2$ (each $<0.00001)$ and $13-15$ y $(0.0001,0.0155)$, respectively. The proportion of low PC or PS patients (19\%) was each higher than that of low AT patients in 7-12 y (0.0005). The number of patients showing both low levels of PC and PS activities were 25 (20\%), 5 of whom had the low levels of all three factors (Supplementary Figure S1 online).

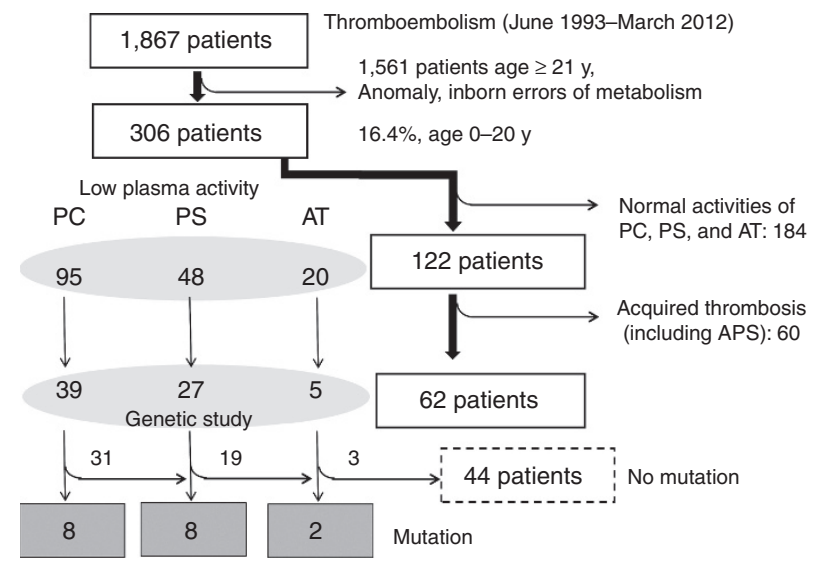

Figure 1. Flowchart of the genetic study of pediatric thrombophilia in a Japanese institution. During the $20 y, 306$ patients ( $\leq 20 y$ of age) were screened by the plasma activities of protein C (PC), protein S (PS), and antithrombin (AT) and underwent the genetic study if they have idiopathic or unusual thrombophilic predispositions.

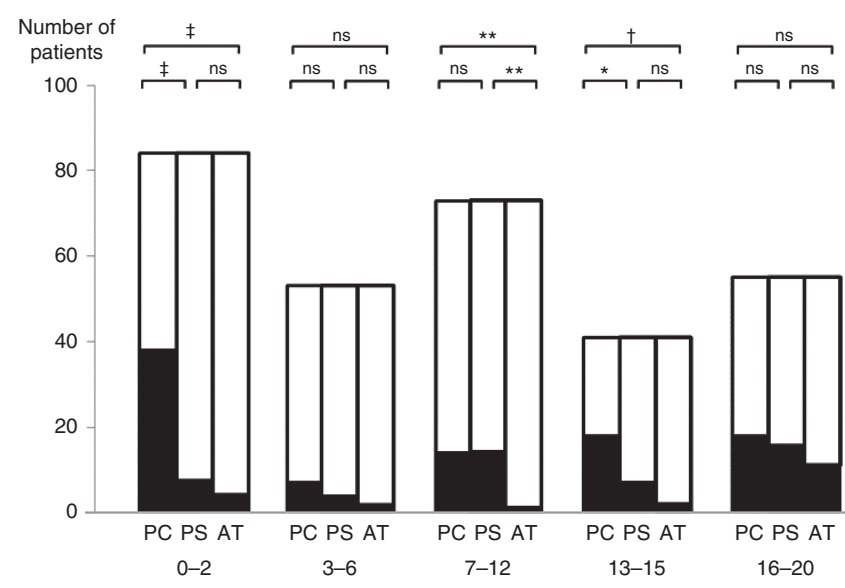

Figure 2. Proportion of patients who showed the low plasma activity of PC, PS, and AT according to the age groups. $0-2$ y: infants ( $n=84$; low PC, $45 \%$; low PS, $8 \%$; low AT, 5\%), 3-6 y: preschoolers ( $n=53$; low PC, $14 \%$; low PS, 8\%; low AT, 4\%), 7-12 y: elementary school children ( $n=73$; low PC, 19\%; low PS, 19\%; low AT, 1\%), 13-15 y: junior high school students ( $n=41$; low PC, 44\%; low PS, 17\%; low AT, 5\%), 16-20y: high school and college or university students ( $n=55$; low PC, 33\%; low PS, 30\%; low AT, $20 \%)$. Closed bar represents the patients who showed each low plasma activity. ${ }^{*} P=0.0155 ;{ }^{*} P=0.0005,{ }^{\dagger} P=0.0001 ;{ }^{\ddagger} P<0.00001$. ns, no statistical significance.

\section{Mutations of PC, PS, and AT Genes in Patients}

Of 62 children who showed the low level of at least one factor, mutation carriers were found in low PC $(n=8 / 39,21 \%)$, PS ( $n=8 / 27,30 \%)$, or AT $(n=2 / 5,40 \%)$ patients, respectively. Forty-four patients carried no mutations. Age distribution of 62 patients differed among three deficiencies (0.0042; Figure 3 ). The proportion of age $0-2$ y patients with PC deficiency (44\%) was the highest among any age group patients with PS or AT deficiency. Six of eight patients with PC gene mutations (75\%) were found in the age group $0-2 \mathrm{y}$, in which only two PS and no AT mutation carriers were determined. Four of eight patients with PS gene mutations (50\%) were found in the age group 7-12 y. No ratio of mutated to analyzed patients differed in the age-groups.

\section{Clinical Onset, Genotype, and Activity Levels of PC, PS, and AT in the Mutation Carriers}

Eighteen patients with a mutation are listed in Table 1. Seven out of eight PC-deficient patients suffered from intracranial thromboembolism and five of them were younger than 2 y. On the other hand, six of eight PS-deficient patients presented in the second decade of life, the onset of which were adult type deep vein thrombosis of pulmonary thromboembolism in five of them. Double allele (homozygous or compound heterozygous) and one allele (heterozygous) mutation(s) were found in half of eight PC-deficient patients, respectively. On the other hand, double allele mutated patients were only two of eight heritable PS-deficient patients, both of whom carried PS-Tokushima. Two other patients had PS-Tokushima. Both patients with heritable AT deficiency carried heterozygote AT mutation. Four of eight patients with PC gene mutation and
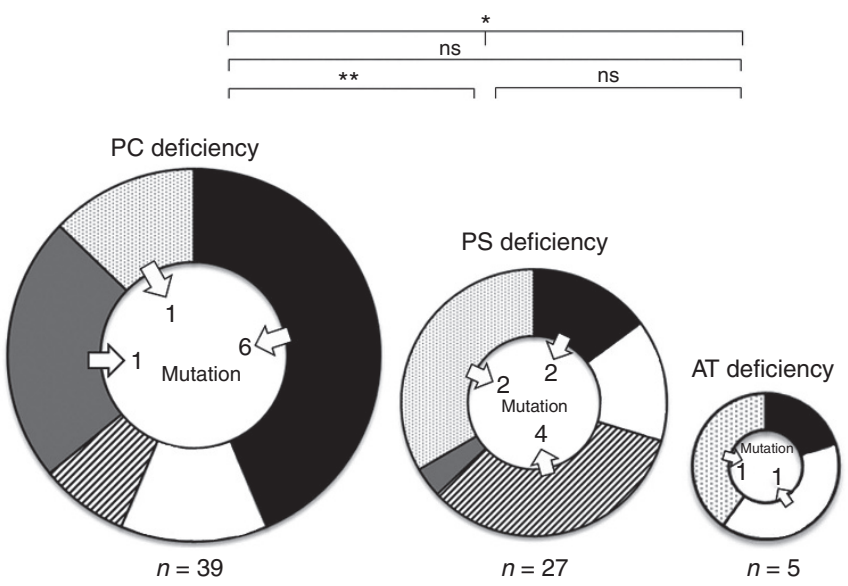

Figure 3. Age distribution of patients with thromboembolism carrying PC, PS, and AT gene mutation. The circle size represents the number of patients. (a) PC deficiency $(n=39)$ : 0-2 y, 44\%; 3-6 y, 13\%; 7-12 y, 8\%; $13-15 y, 23 \%$; and $16-20 y, 13 \%$. Six of eight patients with PC gene mutations (75\%) were found in the age group 0-2 y. (b) PS deficiency $(n=27)$ : $0-2$ y, 15\%; 3-6 y, 15\%; 7-12 y, 33\%; 13-15 y, 4\%; and 16-20 y, 33\%. Four of eight (50\%) patients were found in the age group 7-12 y. (c) AT deficiency $(n=5): 0-2 y, 20 \% ; 3-6 y, 40 \% ; 16-20 y, 40 \%$. All AT gene mutation carriers were found in the age group more than $3 \mathrm{y}$. Closed areas indicate $0-2 \mathrm{y}$ of age, open areas indicate 3-6y of age, hatched areas indicate 7-12 y of age, gray areas indicate 13-15 y of age, and dotted areas indicate 16-20 y of age. ${ }^{*} P=0.0042,{ }^{*} P=0.0019$. ns, no statistical significance. 
Table 1. Plasma activity of protein C, protein S, and antithrombin and each gene mutation in pediatric patients

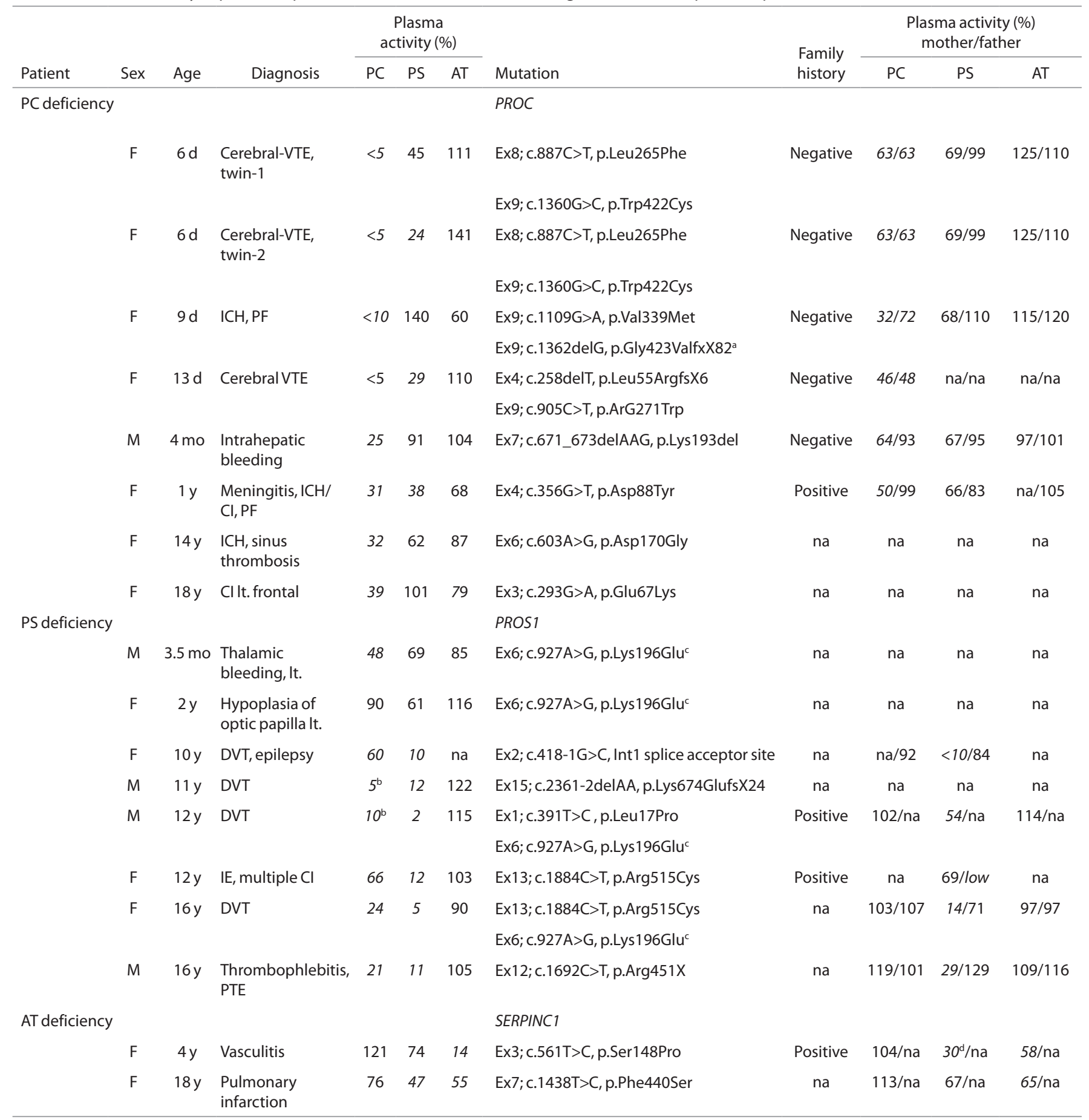

The italic formatting indicates low activity according to the conditional limits of age groups.

$\mathrm{Cl}$, cerebral infarction; DVT, deep vein thrombosis; ICH, intracranial hemorrhage; It., left; na, not assessed; PF, purpura fulminans; PTE, pulmonary thromboembolism; VTE, venous thromboembolism.

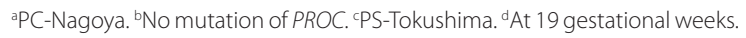

seven of eight patients with PS gene mutation showed reduced activities of PS and PC, respectively.

\section{DISCUSSION}

The first survey on PC, PS, and AT deficiency in Japanese thromboembolic children revealed the contribution to $5.9 \%$ of pediatric thrombosis, and the age-dependent presentation of each PC, PS, or AT deficiency before adult life. Congenital PC and PS deficiencies were the major thrombophilias in Japanese children with different age distribution. Predominant cerebral thromboembolism in PC-deficiency contrasted sharply with extracranial VTE in the other two deficiencies. The 
genotype of PS-deficient children reflected the high prevalence of PS-Tokushima in Japanese general population (1.1-1.8\%; Table 1) $(12,13)$. The Israeli-German cohort studies (14-16) have recently reported the prevalence of congenital deficiencies PC (7.4\%), PS (8.2\%), and AT (6.6\%) in pediatric patients with thromboembolism aged 0.1-18 y. In the cohorts, the proportion of neonates was also higher in PC deficiency (32\%; 8/25 patients) than seen in PS deficiency (10\%; $3 / 30$ patients) or AT deficiency (14\%; $3 / 21$ patients). Half of PC-deficient newborns presented cerebral sinovenous thrombosis and stroke. In this setting, PC-deficient infants characterized the phenotype of pediatric thrombophilias (purpura fulminans and intracranial lesions) beyond the ethnicity (17). The age-specific prevalence and phenotype of pediatric PC deficiency may be useful in the genetic screening for pediatric thrombophilias.

The first concern is the applicability of the lower limits of PC, PS, or AT activity in age groups for the genetic screening. The ranges of anticoagulant activities often vary until the attainment of adult levels, reflecting the individual maturation and vitamin $\mathrm{K}$ status $(18,19)$. Our previous adult study assessed by the same measures reported that half of VTE patients with each low activity carried the heterozygous mutation, which resulted in 33\% positive rate of all patients (20). Miyata et al. (21) confirmed that $32 \%$ of adult VTE patients carried heterozygous mutation of PC, PS, or AT, in the twice sample size, without the screening of plasma activity. In this setting, the concordant mutation rate by the independent studies would warrant the utility of plasma activities for genetic screening in Japanese children. No mutation in $>30 \%$ of patients raises the possibility of large gene deletions, polymorphisms, and the other genetic variations or certain modifiers affecting the activity and antigen concentration (22). The present measures studied polymorphisms including the promoter regions but not deletions. Large deletions are reported to be $3-6 \%$ in these deficiencies (5). Cooper et al. (23) revealed that large deletions make up between 7 and 10\% of PS and AT mutations and only $1 \%$ of PC mutations. The rare occurrence of thrombosis and the wide range of factor activities impeded to define the standard values of "true healthy" children who develop no thromboembolism until the forties. In this study, there was no significant difference in the ratios of mutated patients to the low PC patients who underwent the genetic analysis and in the ratios of mutated patients to the low PS patients analyzed in any age groups. Taking into account high safety margins, the limits of factor activity might be practical for the genetic screening of pediatric thrombophilias.

The proportion, genotype, and first presentation of PC, PS, and AT deficiency during childhood were distinct from those of adult patients. Patients with low PC activity were almost twice the number of those showing low PS activity (Figure 1). On the other hand, the number of patients who carried either PROC or PROS1 mutation was the same eight. The first nationwide survey for pediatric thrombophilia in Japan suggested a higher prevalence of PC deficiency than expected $(24,25)$. The discrepancy may be explained by the wider range of PC activity than that of PS or AT activity in infant and children
$(18,26)$, partly arising from the inherent variation of PC pathway $(27,28)$. Clinical manifestation and thrombin generation differ among the family members having the same PROC mutation (29). Otherwise, absolute PC deficiency rather than PS deficiency may contribute to the development of thromboembolism in the newborn and young infants (30). Half of the PS gene-mutated patients carried PS-Tokushima, two of whom had compound heterozygous mutations with one allele PS-Tokushima (Table 1). Both patients lacked PS activity and developed deep vein thrombosis at 12 and $16 \mathrm{y}$ of age. These findings suggested that high allele frequency of PS-Tokushima made an impact on the genotype of severe PS-deficient children in Japan. By contrast, six of eight patients with PC deficiency presented in infancy, four of whom carried double allele mutation (one PC-Nagoya) having undetectable plasma activity. The genotypes of pediatric PC and PS deficiency corroborated the previous studies on Japanese adult population.

The other concern is the distinct affected site between PC-deficient (intracranial lesion) and PS-deficient children (extracranial VTE). Premature infants with perinatal complications are liable to bleed in the brain. However, intracranial lesions including sinovenous thrombosis were also observed in adolescence (Table 1). The expression levels of endothelial PC receptor and thrombomodulin are constitutively low in the brain compared with other organs. In contrast to the basilar arteries or choroid plexus, intracerebral vascular endothelium does not express thrombomodulin (31). Free PS works as a cofactor of activated PC in the PC pathway. Circulating free PS molecules are relatively high in infancy because of physiologically low C4-binding protein levels (26). It may explain the preferential cerebral lesions in PC-deficient children. Activated PC and thrombomodulin have homeostatic signals critical in regulating coagulation, inflammation, endothelial barrier function, and neuroprotection (32). PC-deficient children might be vulnerable to the brain damage in association with prothrombotic triggers.

One of the limitations in this report was that the results were obtained from a single center study. The number of mutation carriers was small even in the prolonged study. The other limitation was that the consumption effects and/or the plasma replacement therapy might not be completely excluded in assessing the activity levels of the natural anticoagulants. The shorter biological half-life of PC than that of PS or AT might influence the dissociated low activity of PC in infants. However, these findings do not explain the distinct presentation between PC deficiency in infancy and PS deficiency in adolescents or young adults. Multiplex ligation-dependent probe amplification and/or single-nucleotide polymorphism array are needed to search the deletion in the future study. Further comparative study on PC- and PS-deficient infants may shed some light on the pathophysiology and optimal management of pediatric thrombophilias.

In conclusion, PC gene-mutated patients mostly presented with intracranial thromboembolism less than 2 y of age. On the other hand, PS/AT gene-mutated patients were preferentially found in school age children with extracranial VTE. 
The genetic screening of pediatric thrombophilias could be effectively targeted to stroke in low PC infants and deep vein thrombosis in low PS /AT school age children.

\section{METHODS}

\section{Patients and Screening Protocol}

From 1993 to 2012, 1,867 patients with thrombosis in Kyushu University and affiliated institutions were consecutively assessed for thrombophilic predispositions (Figure 1). After the exclusion of patients with anomaly and inborn errors of metabolism, 306 patients aged less than 21 y of age (16.4\%) were enrolled for the study. Clinical information of thromboembolic events and/or coagulopathy was collected from the medical records and the interviews of attending doctors. Of 122 patients showing the low plasma activity, 60 were excluded because of acquired thrombotic disorders including vasculopathy, antiphospholipid syndrome, and autoimmune diseases. Finally, 62 patients underwent the genetic analysis because they had no explainable causes of thromboembolism other than PC, PS, or AT deficiency. Informed consent was obtained from parents of children aged $<21 \mathrm{y}$ of age. This study was certified by the Institutional Review Board of Kyushu University (Fukuoka, Japan; \#232-02, \#448-00).

\section{Coagulation Study}

To exclude the consumption effects at the diagnosis of thromboembolism, the antigen/activity levels of PC, PS, and AT were repeatedly assessed when the clinical conditions were stabilized a couple of weeks to months after the onset of thromboembolism. The measurement of anticoagulation factors were performed as described previously $(20,33)$. Anticoagulant activities of PC and PS were determined using the Staclot PC kit and the Staclot PS kit (Diagnostica Stago, Asnieres, France), respectively. A chromogenic substrate was used to assay for AT activity as heparin-dependent inhibition of bovine thrombin (Chromostrate ATIII kit; Hitachi, Tokyo, Japan). The adult references were first standardized by using pooled normal plasma, in which a level below 3 SD was defined as reduced activity. According to the data in Japanese children $(18,26)$, the lower limits of each activity were defined as follows: in age <90 d: PC 60\%, PS 60\%, and AT $65 \%$ of the adult reference levels (PC 75\%, PS 60\%, and AT $80 \%$ ); 90 d-2 y: PC $85 \%$, PS $85 \%$, AT $65 \%$ of the adult levels; $3-6$ y: each $85 \%$ of adults; and 7-20 y: same as the adults (Supplementary Table S1 online).

\section{Gene Analysis of PC, PS, and AT}

Genomic DNA was extracted from peripheral blood leukocytes. Direct sequencing of PC (PROC exon 1-9), PS (PROS1 exon 1-15), and AT (SERPINC1 exon 1-6) genes was performed as previously described (20). The exon and exon-intron boundary regions of each gene including promoter region were amplified by PCR, and the products were then subjected to direct sequencing using ABI 377 (Perkin Elmer Applied Biosystems, Foster City, CA).

\section{Statistical Analysis}

The median of continuous variables was assessed by the MannWhitney $U$-test. The distribution difference of countable variables was analyzed by chi-square test or Fisher's exact test. $P$ values less than 0.05 were considered significant.

\section{SUPPLEMENTARY MATERIAL}

Supplementary material is linked to the online version of the paper at http:// www.nature.com/pr

\section{ACKNOWLEDGMENTS}

We thank Michiyo Urata, Takeshi Uchiumi (Department of Clinical Chemistry and Laboratory Medicine, Kyushu University Hospital, Fukuoka, Japan), and Naotaka Hamasaki (Department of Clinical Chemistry, Faculty of Pharmaceutical Sciences, Nagasaki International University, Nagasaki, Japan) for the establishment of system, and all the staffs of the Department of Pediatrics, Kyushu University Hospital, Fukuoka, Japan for the clinical managements.

\section{STATEMENT OF FINANCIAL SUPPORT}

This study was supported by a grant from the Kyushu University Clinical Research Network Project and by a grant from the Ministry of Health, Labour and Welfare of Japan.

Disclosure: The authors have no conflict of interests to disclose.

\section{REFERENCES}

1. Spentzouris G, Scriven RJ, Lee TK, Labropoulos N. Pediatric venous thromboembolism in relation to adults. J Vasc Surg 2012;55:1785-93.

2. Nowak-Göttl U, Janssen V, Manner D, Kenet G. Venous thromboembolism in neonates and children-update 2013. Thromb Res 2013;131:Suppl $1:$ S39-41.

3. Tolbert J, Carpenter SL. Common acquired causes of thrombosis in children. Curr Probl Pediatr Adolesc Health Care 2013;43:169-77.

4. Caspers M, Pavlova A, Driesen J, et al. Deficiencies of antithrombin, protein $\mathrm{C}$ and protein $\mathrm{S}$ - practical experience in genetic analysis of a large patient cohort. Thromb Haemost 2012;108:247-57.

5. Roberts LN, Patel RK, Arya R. Venous thromboembolism and ethnicity. Br J Haematol 2009;146:369-83.

6. Shatla HM, Tomoum HY, Elsayed SM, et al. Inherited thrombophilia in pediatric ischemic stroke: an Egyptian study. Pediatr Neurol 2012;47: $114-8$.

7. Sirachainan N, Sasanakul W, Visudibhan A, Chuansumrit A, Wongwerawattanakoon P, Parapakpenjune S. Protein C deficiency in Thai children with thromboembolism: a report of clinical presentations and mutation analysis. Thromb Res 2010;125:200-2.

8. Shen MC, Lin JS, Tsay W. Protein C and protein S deficiencies are the most important risk factors associated with thrombosis in Chinese venous thrombophilic patients in Taiwan. Thromb Res 2000;99:447-52.

9. Tang L, Jian XR, Hamasaki N, et al. Molecular basis of protein S deficiency in China. Am J Hematol 2013;88:899-905.

10. Holzhauer S, Goldenberg NA, Junker R, et al. Inherited thrombophilia in children with venous thromboembolism and the familial risk of thromboembolism: an observational study. Blood 2012;120:1510-5.

11. Sosothikul D, Kittikalayawong Y, Aungbamnet P, Buphachat C, Seksarn P. Reference values for thrombotic markers in children. Blood Coagul Fibrinolysis 2012;23:208-11.

12. Sakata T, Okamoto A, Mannami T, Tomoike H, Miyata T. Prevalence of protein $\mathrm{S}$ deficiency in the Japanese general population: the Suita Study. J Thromb Haemost 2004;2:1012-3.

13. Ikejiri $M$, Wada $H$, Sakamoto $Y$, et al. The association of protein $S$ Tokushima-K196E with a risk of deep vein thrombosis. Int J Hematol 2010;92:302-5.

14. Limperger V, Klostermeier UC, Kenet G, et al. Clinical and laboratory characteristics of children with venous thromboembolism and protein C-deficiency: an observational Israeli-German cohort study. Br J Haematol 2014;167:385-93.

15. Klostermeier UC, Limperger V, Kenet G, et al. Role of protein S deficiency in children with venous thromboembolism. An observational international cohort study. Thromb Haemost 2015;113:426-33.

16. Limperger V, Franke A, Kenet G, et al. Clinical and laboratory characteristics of paediatric and adolescent index cases with venous thromboembolism and antithrombin deficiency. An observational multicentre cohort study. Thromb Haemost 2014;112:478-85.

17. Price VE, Ledingham DL, Krümpel A, Chan AK. Diagnosis and management of neonatal purpura fulminans. Semin Fetal Neonatal Med 2011;16:318-22.

18. Ignjatovic V, Mertyn E, Monagle P. The coagulation system in children: developmental and pathophysiological considerations. Semin Thromb Hemost 2011;37:723-9.

19. Bereczky Z, Kovács KB, Muszbek L. Protein C and protein S deficiencies: similarities and differences between two brothers playing in the same game. Clin Chem Lab Med 2010;48:Suppl 1:S53-66.

20. Kinoshita $S$, Iida $H$, Inoue $S$, et al. Protein $S$ and protein $C$ gene mutations in Japanese deep vein thrombosis patients. Clin Biochem 2005;38:908-15. 


\section{Articles Ichiyamaet al.}

21. Miyata T, Sato Y, Ishikawa J, et al. Prevalence of genetic mutations in protein S, protein C and antithrombin genes in Japanese patients with deep vein thrombosis. Thromb Res 2009;124:14-8.

22. Oudot-Mellakh T, Cohen W, Germain M, et al. Genome wide association study for plasma levels of natural anticoagulant inhibitors and protein $\mathrm{C}$ anticoagulant pathway: the MARTHA project. Br J Haematol 2012;157:230-9.

23. Cooper PC, Goodeve AC, Beauchamp NJ. Quality in molecular biology testing for inherited thrombophilia disorders. Semin Thromb Hemost 2012;38:600-12.

24. Ohga S, Ishiguro A, Takahashi Y, et al.; Japan Childhood Thrombophilia Study Group. Protein C deficiency as the major cause of thrombophilias in childhood. Pediatr Int 2013;55:267-71.

25. Ohga S, Kang D, Kinjo T, et al. Paediatric presentation and outcome of congenital protein C deficiency in Japan. Haemophilia 2013;19: 378-84.

26. Takahashi Y, Yoshioka A. Hemostasis and its regulation system in childhood. Jpn J Pediatr Hematol 1994;8:389-97.

27. Athanasiadis G, Buil A, Souto JC, et al. A genome-wide association study of the protein C anticoagulant pathway. PLoS One 2011;6:e29168.
28. Tang W, Basu S, Kong X, et al. Genome-wide association study identifies novel loci for plasma levels of protein C: the ARIC study. Blood 2010;116:5032-6.

29. Brummel-Ziedins KE, Orfeo T, Callas PW, Gissel M, Mann KG, Bovill EG. The prothrombotic phenotypes in familial protein $\mathrm{C}$ deficiency are differentiated by computational modeling of thrombin generation. PLoS One 2012;7:e44378.

30. Matsunaga Y, Ohga S, Kinjo T, et al. Neonatal asphyxia and renal failure as the presentation of non-inherited protein C deficiency. J Perinatol 2013;33:239-41.

31. Ishii H, Salem HH, Bell CE, Laposata EA, Majerus PW. Thrombomodulin, an endothelial anticoagulant protein, is absent from the human brain. Blood 1986;67:362-5.

32. Wang Y, Zhao Z, Chow N, Ali T, Griffin JH, Zlokovic BV. Activated protein $\mathrm{C}$ analog promotes neurogenesis and improves neurological outcome after focal ischemic stroke in mice via protease activated receptor 1. Brain Res 2013;1507:97-104.

33. Tsuda H, Hattori S, Tanabe S, et al. Screening for aetiology of thrombophilia: a high prevalence of protein $\mathrm{S}$ abnormality. Ann Clin Biochem 1999;36 (Pt 4):423-32. 